Cite this article: Tartaglia GM, Penchev P, Sforza C. Integrating digital technologies for dental prostheses: from impression to single crowns. A pilot study. Stoma Edu J. 2015;2(2):162-167.

\section{INTEGRATING DIGITALTECHNOLOGIES}

FOR DENTAL PROSTHESES: FROM IMPRESSION TO SINGLE CROWNS. A PILOT STUDY

https://doi.org/10.25241/stomaeduj.2015.2(2).art.9

\section{Gianluca Martino Tartaglia ${ }^{1,2}$ Petyo Penchev ${ }^{3}$ Chiarella Sforza ${ }^{1 *}$}

'Functional Anatomy Research Center (FARC) Laboratory of Functional Anatomy of the Stomatognathic Apparatus, Department of Biomedical Sciences for Health, Faculty of Medicine, University of Milan, Milan, Italy ${ }^{2}$ SST Dental Clinic, Segrate (MI), Italy ${ }^{3} 3 \mathrm{~d}$ Object and data Software Taverne, Switzerland

a. DDS, PhD, Assistant Professor and Consultant b. Master Dental Technician c. MD, PhD, Professor
Received: June $1^{\text {st }}, 2015$ Accepted: November $5^{\text {th }}, 2015$

\footnotetext{
* Corresponding author: Prof. Chiarella Sforza Department of Biomedical Sciences for Health, Faculty of Medicine University of Milan, Milan, Italy via Mangiagalli 31 I-20133 Milan, Italy Tel: +390250315385 Fax: +390250315387 e-mail: chiarella.sforza@unimi.it
}

\title{
Abstract
}

Purpose: The aim of this case series was to assess the accuracy of digital workflow for single posterior tooth rehabilitation.

Materials and Methods: Ten patients needing single tooth molar or premolar reconstruction in one or both jaws were randomly selected for this study. The patients were divided into two groups, one treated with the new digital procedure (group A), and a second one with the conventional procedure (group B). The workflow was tested at clinical outcome level to standardize the dental lab, manufacture facility and clinical template.

Results: Ten definitive zirconia-ceramic prostheses were delivered to the patients with one-year encouraging results. The mean overall time analyses for the dental lab revealed differences for group A (40 min) compared to group B (90 min). Total laboratory costs were lower for group A (no cost for traditional delivery and quality control) compared to group B.

Conclusions: Digital workflow creates accurate prostheses, significantly improving efficiencies for the dental team and streamlining the procedures.

Keywords: prosthodontics, digital technologies, digital workflow, single posterior tooth rehabilitation, zirconia-ceramic crowns

\section{Introduction}

The construction of digital dental prostheses both on teeth and implants is an established procedure still subject to optimization from the scientific and professional dental community. ${ }^{1}$ Dental prosthodontics, in particular, derives many advantages from the digital CAD/CAM technology in the production of full-contour reconstruction. ${ }^{2}$ The digital dentistry workflow for prosthetic work is currently still based on an intraoral impression that is subsequently poured in dental stone, notwithstanding that impression materials are subjected to the dimensional changes of the on-going chemical reactions ${ }^{3}$, and stone will show expansion for secondary reactions while setting. ${ }^{4}$ Nevertheless, the traditional workflow is a well consolidated approach in clinical practice and still remains the gold standard, even if research and industry are devoting time and money to develop new digital methods. In particular, digital impressions and $3 d$ printed models are the only two missing steps for the development of the full digital workflow for fixed prosthetic restorations. With a digital impression system, the data from the intraoral scanner can be electronically transmitted to the manufacturer for the fabrication of a definitive prosthetic restoration. With $3 d$ printed models, quality control of dental products can be directly performed from the manufacturing facility lab in terms of checking the inaccuracies resulting from the scanning process, software design, milling, and shrinkage effects. . $^{5}$

The market currently offers different printers with the ability to print various 3D objects using various technologies.

The most commonly used printers are:

O FDM (fusion deposition modeling), where a plastic line is laid down and it builds up objects;

O PBP (Powder based printers), where powder is glued by inkjet;

O SLS (selective laser sintering), where nylon or similar types of thermoplastic powders are melted with a laser beam; O SLA (stereolitography), where UV curable resin is cured in the desired shape by light source.

For dental purposes, stereolithography is the most employed to obtain $3 \mathrm{~d}$ models, 


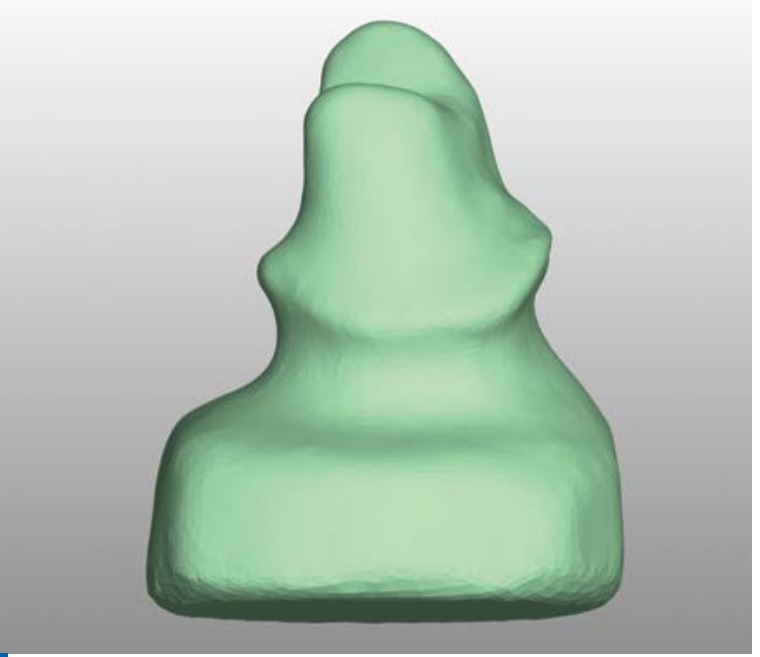

Figure 1a

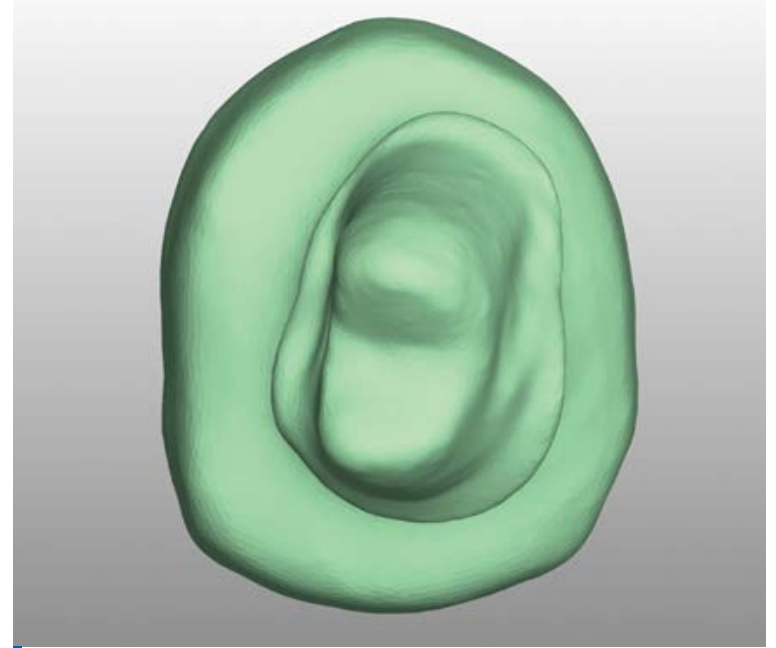

Figure 1c

but sufficient accuracy is still lacking. ${ }^{7}$

The aim of this case series is to present, as a proof-of-principle, a new production concept for milled dental prostheses where inaccuracies are controlled on 3d printed models. ${ }^{8}$ Two groups of patients will be assessed, one treated with the conventional procedure, and a second one with the new digital procedure. Therefore, the clinical outcome of single unit prostheses will be compared between conventional plaster models and $3 d$ printed copies obtained will a full digital workflow. ${ }^{9}$ Additionally, we want to determine whether a $3 d$ printed model can replace the process of traditional plaster casts making and its potential benefits and advantages.

\section{Materials and methods}

Ten patients ( 6 women and 4 men, aged between 31 and 57, average age 43), needing a molar or premolar single tooth (upper or lower) to be restored, were enrolled to receive zirconia crowns from the same private practice between January 2014 and January 2015. Tooth-supported prostheses were used only on root canal treated teeth and teeth with successful endodontic revision.

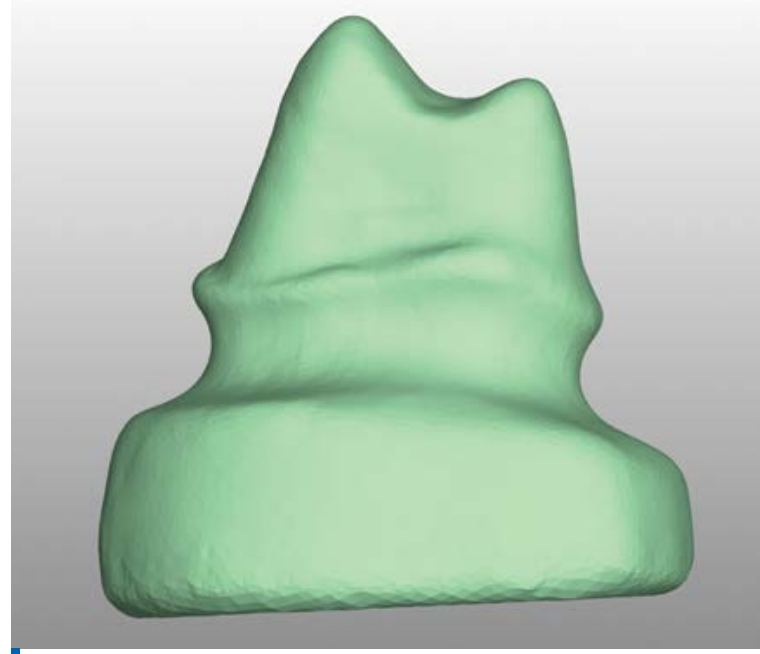

Figure $1 \mathbf{b}$

Figure 1a-b-c. Final appearance of one digital abutment, buccal (A), mesial (B) and occlusal (C) views, just before its $3 D$ printing.

Patient number 1 , tooth 25
All teeth received a prefabricated post. Prior to prosthodontic treatment, all patients underwent a dental hygiene assessment; they all were found to have less than $25 \%$ marginal plaque and no caries. Overall, their oral hygiene was evaluated as good or moderate. Individualized treatment was made to obtain the aforementioned inclusion criteria. Good general health without severe medical or psychological conditions was self-reported by patients. All subjects provided informed consent for the clinical procedures, in accordance with the Declaration of Helsinki and the Italian Law. IRB was unnecessary.

The bone level of the supporting teeth was at least half the root length, and without signs of periodontal or endodontic problems or pathological mobility. The residual coronal tooth structure had a tooth restorability index value equal to or less than 2 . It means that there was no sufficient residual coronal dentine for restoration. ${ }^{10}$ Survival rate was defined as surviving FDPs minus altered FDPs based on two (grades 2 and 3) of the three grades scale of chipping fractures. Surface chipping is graded 1 if the fractured surface is not extended into a functional area and polishing is 


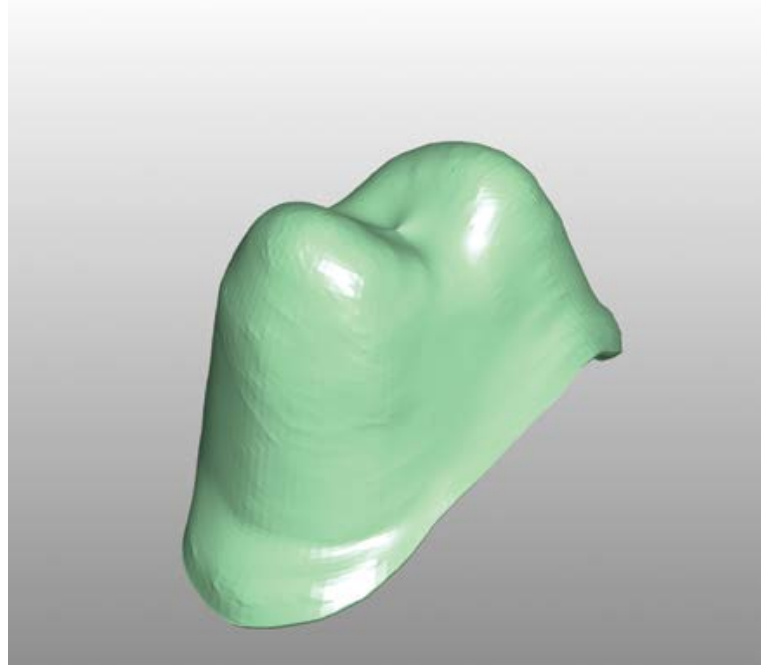

Figure 2. Final appearance of one digital zirconia core, just before its 3D milling. Patient number 1 , tooth 25

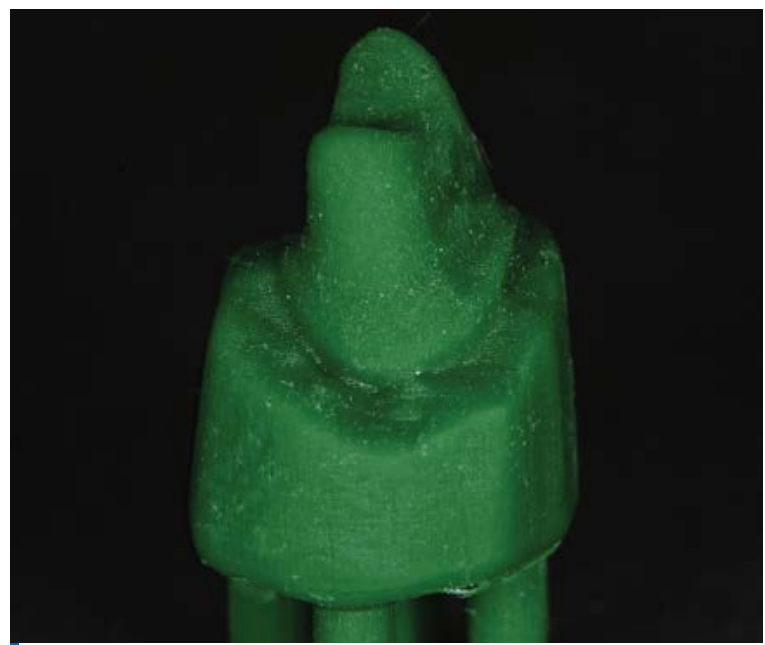

Figure 4. The printed abutment. Patient number 1 , tooth 25

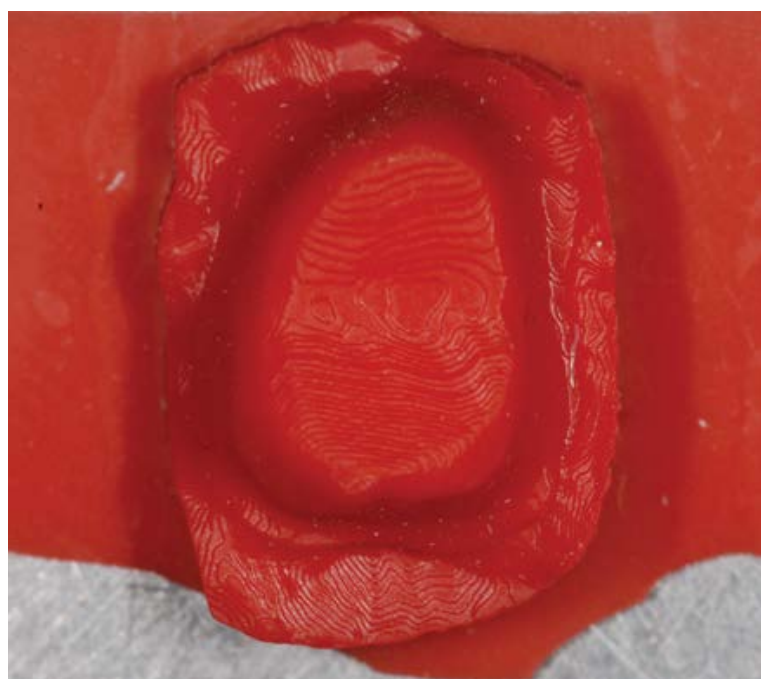

Figure 6. Magnification of the occlusal surface of one printed abutment (original magnification $4 X)$. Patient number 2, tooth 16

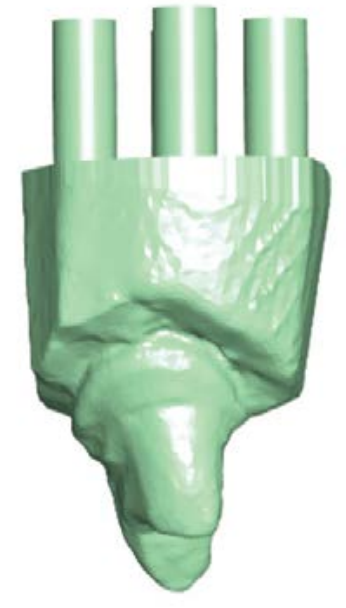

Figure 3. Final appearance of one digital abutment prepared for $3 \mathrm{~d}$ printing. Patient number 1 , tooth 25

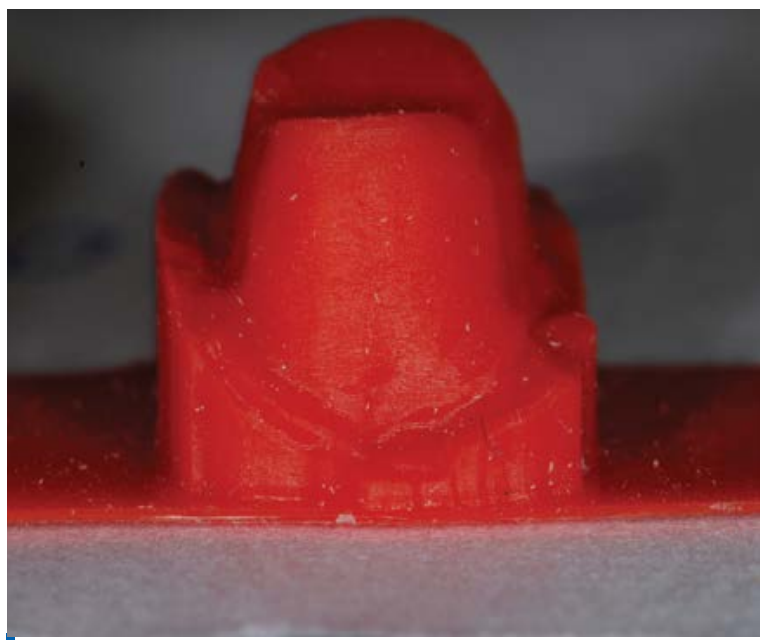

Figure 5. Magnification of the buccal surface of one printed tooth abutment (original magnification 2X). Patient number 2, tooth 16

possible. Recontouring will result in an acceptable alteration of the anatomic form from the original anatomy. ${ }^{11}$ After the abutment teeth preparations were completed, the chairside interim restorations were fabricated and inserted with interim cement according to Tartaglia et al. ${ }^{12}$ The patient was asked to return after 2 weeks to allow time to evaluate both esthetics and function before the definitive impression appointment.

The double-cord technique was used for soft tissue management during definitive impression making with polyether (Impregum/Permadyne, 3M ESPE AG, Seefeld, Germany) in a customized tray (Apex trays, Megadenta Dentalprodukte Radeberg, Germany). Interocclusal record registration was performed with rigid wax (Moyco Dental wax, Miltex, USA). The plaster models were prepared and digitized to identify and mark crown margins, virtual ditching and articulation. The crowns were designed from the digital library of the dental lab with minor modifications by the dental technician. 


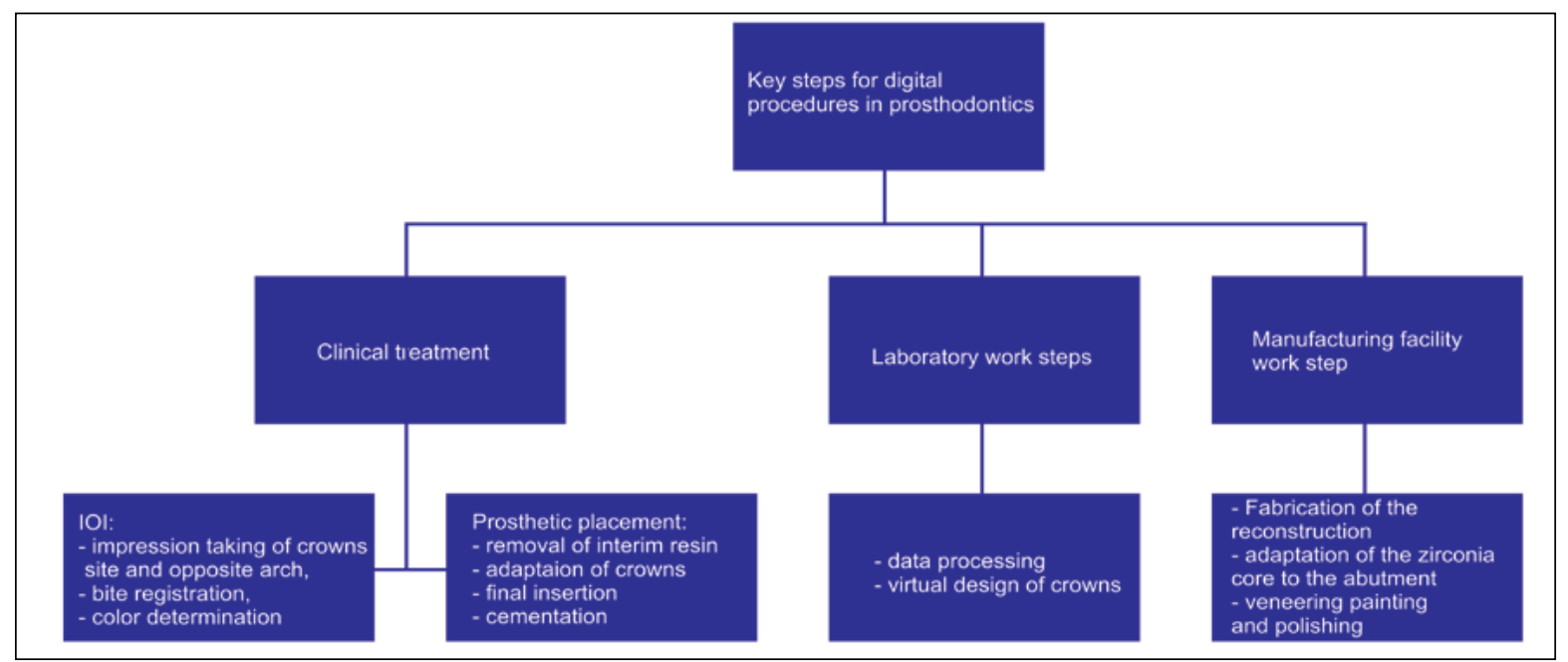

Table 1: Key steps for the new digital procedures in prosthodontics (IOI: intraoral impression)

The completed scan data were digitally delivered to a manufacturing facility (3d Objects and Data Software, Taverne, Switzerland).

The manufacture of $3 d$ printed models from the plaster casts scanning used an STL file where approximately 350,000 vertices and 600,000 faces were depicted; file size was approximately 30-35 $\mathrm{MB}$. The resolution of the scanning (and of the obtained model too) was $10 \mu \mathrm{m}$. The resolution of the $3 d$ printer was approximately between 0 to 50 $\mu \mathrm{m}$, so no reduction and re-mesh of the digitized models was necessary. A trained operator digitally modified the file with particular attention to selfintersecting or duplicated faces, non-manifold edges and vertices filling of the holes, thus rendering the model ready and fully valid for the $3 d$ printer.

Finally, the STL model was sliced into individual layers, the path of printing nozzle was computed and the STL file was converted to GCODE file by Slic $3 r$ and printed.

At the same time, the plaster models were sent to the manufacturer by traditional delivery. The new individually zirconia CAD/CAM core was milled in the presintered state (Zirite, Keramo, Tavernerio, Como, Italy) and subsequently sintered in accord with the manufacturer. Feldspathic porcelain (CZR Noritake Kizai Co. Ldt., Nagoya, Japan) was fused on the core with zirconium oxide margins by one master ceramist in accordance with a slow cooling protocol. ${ }^{13}$ From the manufacturer the milled crowns were randomly assigned to group A with fabrication steps controlled on $3 d$ printed model, and group B with procedures controlled on traditional plaster models. The trial insertion of the milled restorations was completed in the sintered state to allow verification of the marginal fit and internal adaptation. Each fabrication step was evaluated to control prosthesis accuracies, occlusal function and esthetic results. At the end of the process, dental prostheses and traditional models were returned to the dental laboratory and consequently to the dentist.

At the insertion appointment, the marginal adaptation and restoration fit were verified with a polyvinyl siloxane material (Fit Checker Black; GC America, Alsip, IL, USA). By using 8-mm-wide, 8-mm-thick shimstocks (Hanel, Roeko, Langenau, Germany), proximal contact points and occlusal contacts were adjusted as necessary and tested in maximum intercuspation with no interferences in lateral excursions. Final crowns polishing and luster prior of insertion were achieved by using pearl surface paste (Noritake Kizai Co. Ldt., Nagoya, Japan). The abutment teeth were cleaned before cementation, that was made using a glass-ionomer cement (Ketac, 3M ESPE AG, Seefeld, Germany).

A functional analysis of masticatory muscles was made in all patients just before and after the cementation of the final prostheses. ${ }^{14}$ The residual cement was removed and post insertion home care instructions were provided to the patient. Periapical radiographs were used to confirm the complete seating of definitive restorations and the removal of residual luting agent. The patients were also enrolled in a hygiene program with 6-month intervals and demonstrated no complications or failure during the 12-month follow-up period.

At a recall appointment, all the patients were visited by an independent operator that had not been involved in the original prosthetic procedures and was not aware of the group the patient belonged to (group A, fabrication steps controlled on 3d printed model; group $B$, procedures controlled on traditional plaster models). Follow-up time ranged between 11 and 13 months (median: 12 months).

\section{Results}

Table 1 summarizes the key steps for the new digital workflow in prosthodontics. As an example of data processing and virtual design of the crowns, figures 1-3 show the CAD steps for a single tooth prosthesis (tooth 25) in patient number 1 (a woman aged 41 years). The printed abutment is illustrated in fig. 4. Details of the buccal and occlusal surfaces of the printed abutments are shown in figures 5 and 6 (single tooth prosthesis, tooth 16 in patient number 2, a woman aged 51 years).

As primary outcome, economic analyses with time assessment and cost calculations for prosthetic 
treatment as well as laboratory work steps were performed and compared. All digitally generated crowns required no interproximal or occlusal adaptations. In addition, the clinical treatment could be managed during two appointments for (1) intraoral impression and (2) prosthetic placement, with a mean total chair time of $20 \mathrm{~min}$ per patient/reconstruction. The mean overall time analyses for the dental lab revealed differences for group A (40 min) compared with group B (90 min). Total laboratory costs were lower for group A (no cost for traditional delivery and quality control) compared with group $B$. The advantages for the dental lab could be summarized in:

O lower price in terms of quality control costs;

O reduced traditional working time;

O property maintenance of the prosthodontic project.

At the 12 months follow-up visit, all patients were evaluated, and no complications or failures were recorded. The mean White Esthetic Scores (WES) ${ }^{15}$ were comparable within the two groups. Patient's satisfaction was high in all cases.

\section{Discussion and Conclusions}

Among the wishes of dental practitioners there is the interest to offer the advantages of oral rehabilitations to more patients. This can be accomplished by reducing the overall clinical treatment cost and time used, and the total amount of technical production process to achieve

\section{Bibliography}

1. Beuer F, Stimmelmayr M, Gueth JF, Edelhoff D, Naumann $M$. In vitro performance of full-contour zirconia single crowns. Dent Mat. 2012;28(4):449-456.

2. Patel N. Integrating three-dimensional digital technologies for comprehensive implant dentistry. J Am Dent Assoc. 2010;141(Suppl 2):20S-24S.

3. Johnson GH, Craig RG. Accuracy of four types of rubber impression materials compared with time of pour and a repeat pour of models. J Prosthet Dent. 1985;53(4):484-490.

4. Millstein PL. Determining the accuracy of gypsum casts made from type IV dental stone. J Oral Rehabil. 1992;19(3):239-243. 5. Denry IL, Holloway JA, Rosenstiel SF. Effect of ion exchange on the microstructure, strength, and thermal expansion behavior of a leucite reinforced porcelain. J Dent Res. 1998;

77(4):583-588.

6. Walton TR. An up to 15-year longitudinal study of 515 metal-ceramic FPDs: Part 1. Outcome. Int J Prosthodont. 2002;15(5):439-445.

7. Keating AP, Knox J, Bibb R, Zhurov Al. A comparison of plaster, digital and reconstructed study model accuracy. J Orthod. 2008;35(3):191-201; discussion 175.

8. Bindl A, Mörmann WH. Marginal and internal fit of allceramic CAD/CAM crown copings on chamfer preparations. J Oral Rehabil. 2005;32(6):441-447.

9. Christensen GJ. Marginal fit of gold inlay castings. J Prosthet Dent. 1966;16(2):297-305.

10. Bandlish RB, McDonald AV, Setchell DJ. Assessment of the amount of remaining coronal dentine in root-treated teeth. $J$ a reasonable cost-benefit ratio in combination with high quality and precision of the prosthetic reconstructions. ${ }^{16-17}$ Technical development in the field of digital dental medicine has opened this opportunity. But new competences are requested to dental operators to achieve the entire fabrication digital dental process, starting clinically, following digital designing avoiding the risk to delegate the prosthodontic project to a third part, and last but not least assuming all the competence the digital process is requesting. The findings of this clinical case series revealed that this a feasible treatment concept in the digital workflow in prosthesis manufacture. Dental practitioners can offer a streamlined treatment approach for single-tooth replacement, at least for posterior sites.

Clinicians, dental technicians and patients would even benefit more from these procedures because manufacture only should be delegated to production centers.

The prosthesis design will remain in the dental clinic and lab, avoiding the delegation of the prosthodontic project to third parts. A more patient-centered outcome will be obtained.

Of course, the current data were limited to single unit posterior crowns, and the procedure should be tested for more complex restorative procedures. Obviously further large-scale studies with long-term follow-up observations are necessary to investigate the clinical performance of the treatment concept.
Dent. 2006:34(9):699-708.

11. Anusavice KJ. Standardizing failure, success, and survival decisions in clinical studies of ceramic and metal-ceramic fixed dental prostheses. Dent Mater. 2006;28(1):102-111.

12. Tartaglia GM, Sidoti E, Sforza C. Seven-year prospective clinical study on zirconia-based single crowns and fixed dental prostheses. Clin Oral Investig. 2015;19(5):1137-45.

13. Guazzato M, Walton TR, Franklin W, Davis G, Bohl C, Klineberg I. Influence of thickness and cooling rate on development of spontaneous cracks in porcelain/zirconia structures. Aust Dent J. 2010;55(3):306-310.

14. Ferrario VF, Tartaglia GM, Galletta A, Grassi GP, Sforza C. The influence of occlusion on jaw and neck muscle activity: a surface EMG study in healthy young adults. J Oral Rehabil. 2006;33(5):341-348.

15. Belser, UC, Grüter, L, Vailati, Bornstein, MM, Weber, HP, Buser, D. Outcome evaluation of early placed maxillary anterior single- tooth implants using objective esthetic criteria: a crosssectional, retrospective study in 45 patients with a 2- to 4-year follow-up using pink and white esthetic scores. J Periodont. 2009;80(1):140-151

16. Brägger $U$, Krenander $P$, Lang NP. Economic aspects of single-tooth replacement. Clin Oral Implants Res. 2005; 16(3):335-341.

17. Bouchard P, Renouard F, Bourgeois D, Fromentin O, Jeanneret $M H$, Beresniak $A$. Cost-effectiveness modeling of dental implant vs. bridge. Clin Oral Implants Res. 2009;20(6):583-587. 


\section{Gianluca Martino Tartaglia, DDS, PhD \\ DDS, PhD, Assistant Professor and Consultant, Department of Biomedical Sciences for Health, Faculty of Medicine, University of Milan, Milano, Italy}

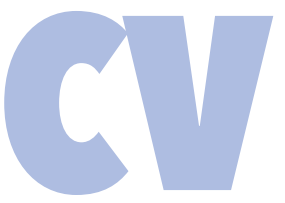

Dr. Gianluca Martino Tartaglia received his D.D.S. degree from the University of Milan School of Dentistry, Italy in 1991, and his Ph.D. in

Morphological Sciences from the same university in 1996.

He is currently a staff member of the Laboratory of Functional Anatomy of the Stomatognathic Apparatus, and he works in private practice in Milan.

$\mathrm{He}$ is the author of more than 100 international research papers on the stomatognathic system. In particular, he is an expert in the surface electromyography of masticatory and neck muscles.

\section{0ugstons}

\section{The most used printers for dental prostheses are:}

$\square$ a. PBP (Powder based printers), where powder is glued by inkjet;

b. SLA (stereolitography), where UV curable resin is cured in the desired shape by light source;

c. SLS (selective laser sintering), where nylon is melted with laser beam;

$\square \mathrm{d}$. FDM (fusion deposition modeling), where a plastic line is laid down and builds up objects.

\section{In the current investigation:}

$\square$ a. Ten patients received one single crown prosthesis each;

$\square$ b. One patient received 10 single crown prostheses;

$\square$ c. Five patients received a full arch rehabilitation;

$\square$ d. Ten patients received one multiple unit prosthesis for anterior teeth.

\section{To manufacture the $3 \mathrm{~d}$ printed models we used}

$\square$ a. JPEG files with RGB color conversion;

b. STL files with approx 100 vertices and 60 faces ;

c. TIFF files with CMYK color conversion;

$\square$ d. STL files with approx 350,000 vertices and 600,000 faces.

\section{The mean overall time analyses for the dental laboratory was:}

a. $100 \%$ longer in the group with procedures controlled on traditional plaster models;

b. $80 \%$ longer in the group with procedures controlled on $3 \mathrm{~d}$ printed model;

c. independent from the kind of procedure control;

$\square$ d. $40-50 \%$ longer in the group with procedures controlled on traditional plaster models. 